\title{
REVIEW
}

\section{Crosstalk between the oral microbiota, mucosal immunity, and the epithelial barrier regulates oral mucosal disease} pathogenesis

\author{
Dongjia Lin ${ }^{1}$, Lisa Yang ${ }^{1}$, Liling Wen ${ }^{1}$, Huanzi Lu' ${ }^{1}$, Qianming Chen ${ }^{2}$ and Zhi Wang (D)
}

Oral mucosal disease (OMD), which is also called soft tissue oral disease, is described as a series of disorders or conditions affecting the mucosa and soft tissue in the oral cavity. Its etiology is unclear, but emerging evidence has implicated the influence of the composition of the oral mucosa and saliva-resident microbiota. In turn, this dysbiosis effects the immune response balance and epithelial barrier function, followed by the occurrence and progression of OMD. In addition, oral microbial dysbiosis is diverse in different types of diseases and different disease progressions, suggesting that key causal pathogens may exist in various oral pathologies. This narrative literature review primarily discusses the most recent findings focusing on how microbial dysbiosis communicates with mucosal adaptive immune cells and the epithelial barrier in the context of five representative OMDs, including oral candidiasis $(\mathrm{OC})$, oral lichen planus (OLP), recurrent aphthous ulcer (RAU), oral leukoplakia (OLK), and oral squamous cell carcinoma (OSCC), to provide new insight into the pathogenetic mechanisms of OMDs.

Mucosal Immunology (2021) 14:1247-1258; https://doi.org/10.1038/s41385-021-00413-7

\section{INTRODUCTION: CROSSTALK BETWEEN THE ORAL MICROBIOTA, MUCOSAL IMMUNITY AND THE EPITHELIAL BARRIER IN ORAL PATHOLOGIES}

Oral mucosal disease (OMD), which is also called soft tissue oral disease, is described as a series of disorders or conditions affecting the mucosa and soft tissue in the oral cavity. OMD mainly includes oral infectious diseases, with oral candidiasis (OC) as the representative; ${ }^{1}$ oral mucosal patches striae diseases, with oral lichen planus (OLP) as the representative; ${ }^{2}$ ulcerative lesions of the oral mucosa, with recurrent aphthous ulcer (RAU) as the representative; ${ }^{3}$ oral premalignancy, with oral leukoplakia $(\mathrm{OLK})^{4}$ as the representative; oral cancer and neoplasms, with oral squamous cell carcinoma $(\mathrm{OSCC})^{4}$ as the representative etc. (Table 1$).^{5-10}$ The etiopathogenesis of OMDs is complicated and not fully understood. Diverse factors, including genetic predisposition, immunologic disturbances, viral and bacterial infections, food allergies, vitamin and microelement deficiencies, hormonal imbalance, mechanical injuries, and stress, have been suggested to be associated with OMDs. ${ }^{1-3}$ Interestingly, all these factors disrupt the diversity and composition of the commensal oral microbiota. Dysbiosis commonly describes a compositional and functional alteration in the microbiota that is driven by a set of environmental and host-related factors that perturb the microbial ecosystem to an extent that exceeds its resistance and resilience capabilities. ${ }^{11}$ Here, we use the extended definition of dysbiosis, namely, a microbial community state that is not only statistically associated with a disease but also functionally contributes to the etiology, diagnosis, or treatment of the disease (Fig. 1). ${ }^{12}$ Recent studies indicate that oral microbial dysbiosis is diverse in different types of diseases ${ }^{13-16}$ and changes during disease progression, ${ }^{14,17-19}$ suggesting that key causal pathogens may exist in various oral lesions. However, the role of the oral microbiota in inducing or progressing oral pathologies has not been thoroughly characterized.

Oral microbiota dysbiosis may cause diseases through several molecular mechanisms. Most recently, a study has demonstrated that finely tuned crosstalk between the oral microbiota, immune cells, and the epithelium is critical for the maintenance of the mucosal architecture and homeostasis. ${ }^{20-24}$ An increasing body of evidence suggests that perturbations of the mucosal microbiota can modulate innate and adaptive immune responses, with inflammation arising due to a reduction in the number of symbiont microorganisms and/or an increase in the number of pathobiont microorganisms (commensal bacteria with pathogenic potential). ${ }^{25-28}$ For example, one mechanism by which these microbes regulate immunity is by controlling regulatory $T$ cells (Tregs) and $T$ helper 17 (Th17) cells. ${ }^{29-31}$ In addition, the epithelium recognizes and responds to the microbiota, and in turn, microbial dysbiosis and associated metabolite alterations destroy the integrity of the mucosal epithelium and its barrier functions. ${ }^{23,32}$ Given that the entire community of microbial residents influences immune response balance ${ }^{33,34}$ and epithelial barrier function, ${ }^{23,35}$ we argue that OMDs can potentially be the outcome of dysbiosis due to homeostatic host-microbe interaction breakdown. The interplay between the intestinal microbiota, immune system and epithelial barrier has been well discussed, ${ }^{21,23,32,33}$ but considerable gaps in our knowledge of the oral cavity remain. In this narrative review, we highlight the

\footnotetext{
${ }^{1}$ Hospital of Stomatology, Guanghua School of Stomatology, Guangdong Provincial Key Laboratory of Stomatology, Sun Yat-Sen University, Guangzhou, Guangdong, PR China

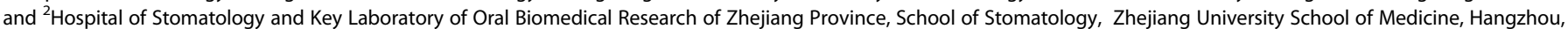
Zhejiang, PR China

Correspondence: Zhi Wang (wangzh75@mail.sysu.edu.cn)
}

Received: 16 January 2021 Revised: 26 April 2021 Accepted: 4 May 2021 Published online: 26 May 2021 
Table 1. Classification of oral mucosal disease.

\begin{tabular}{|c|c|}
\hline Classification & Representative diseases \\
\hline \multirow[t]{3}{*}{ Oral infectious diseases, OID } & Oral candidiasis, $\mathrm{OC}^{\mathrm{a}}$ \\
\hline & Herpes simplex, HS \\
\hline & Coccigenic stomatitis, CS \\
\hline \multirow{3}{*}{$\begin{array}{l}\text { Oral mucosal patches striae } \\
\text { diseases, OMPSD }\end{array}$} & Oral lichen planus, OLPa \\
\hline & Leukokeratosis \\
\hline & Discoid lupus erythematosus, DLE \\
\hline \multirow{3}{*}{$\begin{array}{l}\text { Ulcerative lesions of the oral } \\
\text { mucosa, ULOM }\end{array}$} & Recurrent aphthous ulcer, $\mathrm{RAU}^{\mathrm{a}}$ \\
\hline & Behcet's disease, BD \\
\hline & $\begin{array}{l}\text { Traumatic mucosal hematoma and } \\
\text { traumatic ulceration, TMH \& TU }\end{array}$ \\
\hline \multirow[t]{3}{*}{ Oral premalignancy, OPM } & Oral leukoplakia, OLK ${ }^{\mathrm{a}}$ \\
\hline & Oral erythroplakia, OEK \\
\hline & Oral submucous fibrosis, OSF \\
\hline \multirow[t]{3}{*}{$\begin{array}{l}\text { Oral cancer and } \\
\text { neoplasms, OCN }\end{array}$} & $\begin{array}{l}\text { Oral squamous cell carcinoma, } \\
\text { OSCC }^{\mathrm{a}}\end{array}$ \\
\hline & Basal Cell Carcinoma \\
\hline & Malignant Melanoma \\
\hline \multirow[t]{3}{*}{ Allergic stomatitis, AS } & $\begin{array}{l}\text { Allergic medicamentosus } \\
\text { stomatitis, AMS }\end{array}$ \\
\hline & Contacted stomatitis, CS \\
\hline & Angioneurotic edema, $\mathrm{AE}$ \\
\hline \multirow{3}{*}{$\begin{array}{l}\text { Bullous oral mucosal } \\
\text { diseases, BOMD }\end{array}$} & Pemphigus \\
\hline & $\begin{array}{l}\text { Mucous membrane } \\
\text { pemphigoid, MMP }\end{array}$ \\
\hline & Linear $\lg A$ disease, LAD \\
\hline \multirow[t]{3}{*}{ Orofacial granulomatosis, OFG } & $\begin{array}{l}\text { Melkersson-Rosenthal } \\
\text { syndrome, MRS }\end{array}$ \\
\hline & Granulomatous cheilitis, GC \\
\hline & Sarcoidosis \\
\hline \multirow[t]{3}{*}{ Labiolingual diseases, LD } & Cheilitis \\
\hline & Lingual papillitis \\
\hline & Burning mouth syndrome, BMS \\
\hline \multirow[t]{3}{*}{$\begin{array}{l}\text { Oral manifestations of other } \\
\text { diseases }\end{array}$} & $\begin{array}{l}\text { Acquired immune deficiency } \\
\text { syndrome, AIDS }\end{array}$ \\
\hline & Sjögren's syndrome, SS \\
\hline & Kawasaki disease, KD \\
\hline
\end{tabular}

characteristics of the microbial composition of different OMDs and explore the potential causal pathogens and biomarkers in various oral pathologies. We also focus on the interactions between oral microbes, adaptive immune cells and the epithelial barrier and how these communications influence the following five representative OMDs: OC, OLP, RAU, OLK, and OSCC.

\section{THE ROLE OF CANDIDA ALBICANS IN ORAL CANDIDIASIS}

OC is commonly referred to as "thrush" and is the most common opportunistic fungal infection that generally affects the oral mucosa. The main causative agent, i.e., Candida albicans ( $C$. albicans), is a highly versatile commensal organism that is well adapted to its human host; however, changes in the host microenvironment can promote the transition from commensalism to pathogenesis. ${ }^{1,13}$ This transition heavily relies on an impressive repertoire of virulence factors, most notably including cell surface adhesins, proteolytic enzymes, morphologic switching, and drug resistance development. ${ }^{1}$ Since a clear understanding of the pathogenesis mechanisms of $\mathrm{OC}$ is currently lacking, we review the role of $C$. albicans and its interplay with the host adaptive immune response and mucosal barrier.

C. albicans shapes the host oral microbiota in oral candidiasis C. albicans is by far the main causative agent of OC and account for up to $95 \%$ of cases. The history of the identification of the etiological agent of OC has been fully described in previous studies. ${ }^{1}$ However, no data concerning the species distribution among OC patients were available until a recent large-scale population-based study discovered that of 11,161 isolated Candida strains, C. albicans remained the most common species (75.37\%), followed by Candida tropicalis (C. tropicalis) $(6.06 \%)$, Candida krusei (C. krusei) (2.79\%), and Candida glabrata (C. glabrata) (2.02\%). Surprisingly, both the proportion and number of C. glabrata isolates dramatically increased over the 4 consecutive years of the study. ${ }^{13}$

C. albicans is not only an important component of the oral microbiota but also an important player in communication in the oral microbiome. In a healthy host, unperturbed commensal bacterial communities are crucial for limiting $C$. albicans colonization at mucosal sites. ${ }^{36}$ When the microbial equilibrium is changed by immunosuppression, certain bacterial species may overgrow and form mutualistic relationships with $C$. albicans. The direct or indirect interactions between C. albicans-bacterial cells have been recently reviewed. ${ }^{1,37}$ These studies suggest that in the oral cavity, the coadhesion of $C$. albicans with bacteria is essential for $C$. albicans persistence; therefore, these interactions may enhance colonization in the host. ${ }^{38}$ Moreover, in turn, the pathogen $C$. albicans may lead to well-coordinated dysbiosis, which amplifies mucosal damage. Increasing evidence suggests that $C$. albicans induces mucosal bacterial dysbiosis, which promotes invasive infection. ${ }^{39}$ However, the influence of Candida populations on the microbial community composition is not understood. Future studies should consider OC pathogenesis integrally related to the physiology of the resident microbial communities within which $C$. albicans resides as a commensal or cause of disease.

Host adaptive immune response to $C$. albicans in oral candidiasis As the oral mucosa is frequently colonized, the host immune response in the oral cavity is oriented toward a more tolerogenic state; therefore, local innate immune defenses play a central role in maintaining Candida in its commensal state. ${ }^{1,40}$ Several comprehensive reviews of the innate immune response during C. albicans mucosal infection have been recently published. ${ }^{1,40,41}$ Here, we emphasize the local oral adaptive immune defenses that play a vital role in the defense response against Candida in its pathogenic state during $O C$.

The Th17-type adaptive immune response is mainly involved in mucosal host defenses by controlling the initial growth of Candida and inhibiting subsequent tissue invasion. Recent studies have elucidated the overwhelming role of the Th17/interleukin (IL)-17 axis in protection against candidiasis, which is mainly caused by $C$. albicans. ${ }^{42-47}$ First, C. albicans epitopes can activate STAT3, which is necessary for Th17 proliferation and function, through secondary mediators, ensuring initial pattern recognition and providing a cytokine environment for the activation of Th17 responses. $^{48}$ IL-17 is produced within $1-2 \mathrm{~d}$ by $\mathrm{CD}^{+}{ }^{+} \mathrm{CD} 4{ }^{+} \mathrm{CD} 44 \mathrm{hiTCR}^{+}{ }^{+} \mathrm{CRR} 6^{+}$natural Th17 (nTh17) cells and tongue-resident populations of $\gamma \delta \mathrm{T}$ cells but not $\mathrm{T}$ cell receptor (TCR)-deficient innate lymphoid cells (ILCs) or natural killer (NK) cells. ${ }^{43-46}$ IL-17 promotes granulopoiesis and neutrophil accumulation in peripheral tissues for pathogen clearance and host defense against Candida infections. ${ }^{47}$ A previous study discovered that mono-genetic mutations of stat3 associated with the loss of Th17 cells could enhance acute oropharyngeal candidiasis $(\mathrm{OPC}){ }^{49}$ Mice and humans lacking IL-17R experience chronic mucosal candidiasis. ${ }^{45}$ Anti-IL-17A antibodies, which neutralize IL- 
a Oral homeostasis

\section{b Oral dysbiosis}

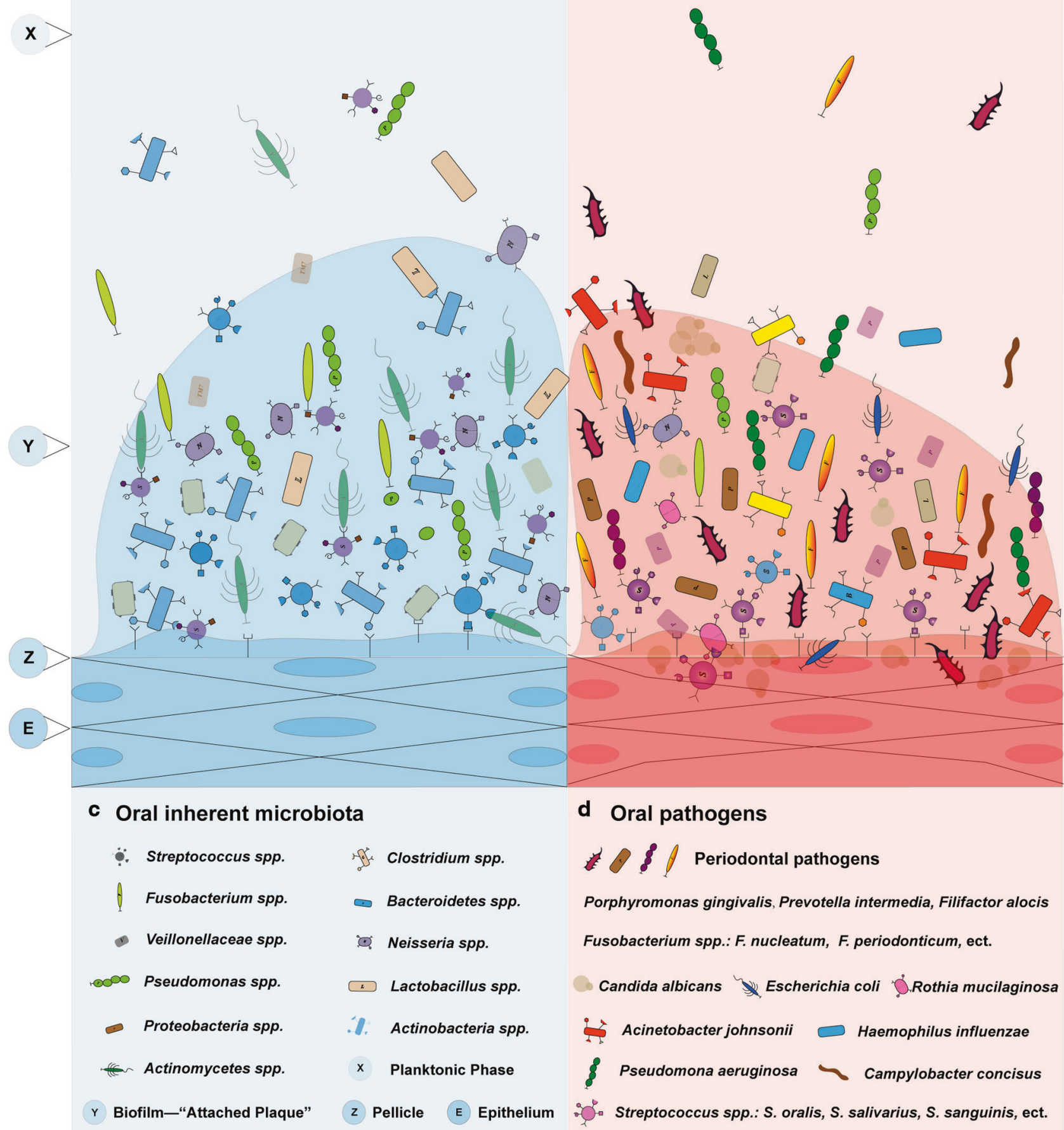

Fig. 1 Oral microbiota homeostasis and dysbiosis. a The oral cavity comprises $\sim 700$ bacterial phyla categorized into six major phyla. The highly associated microorganisms of oral cavity appear sequentially and maintain the homeostasis to keep the oral cavity healthy. $\mathbf{b}$ Dysbiosis is a microbial community state that is not only statistically associated with a disease but also functionally contributes to the etiology, diagnosis, or treatment of the disease. c Oral inherent microbiota. d Oral common pathogens that cause diseases.

$17 \mathrm{~A}$ and IL-17AF, caused elevated oral fungal loads in a mouse model of acute OPC. ${ }^{50}$ Second, $\mathrm{CD}^{+} \mathrm{CD}_{25} 5^{+}$Foxp $3^{+}$Tregs play crucial immunomodulatory roles during infection..$^{31,34,51,52}$ In the context of mouse OPC, these cells mediate increased protection from apoptosis during the late phase of infection and reinfection. Tregs undergo reduced cell death because they are refractory to TCR restimulation-induced cell death (RICD). The enhanced viability depends on increased transforming growth factor- $\beta 1$ (TGF- $\beta 1$ ) signaling, which results in the upregulation of cellular FADD-like IL-1 $\beta$-converting enzyme (FLICE)-inhibitory protein (CFLIP) in Tregs. Protection from cell death is abrogated in the absence of TGF- $\beta 1$ signaling in Tregs during OPC. ${ }^{52}$ During this process, Treg cells induce IL-17 cytokines in responding CD4 ${ }^{+}$ (Tresp) cells, which markedly enhances fungal clearance and 


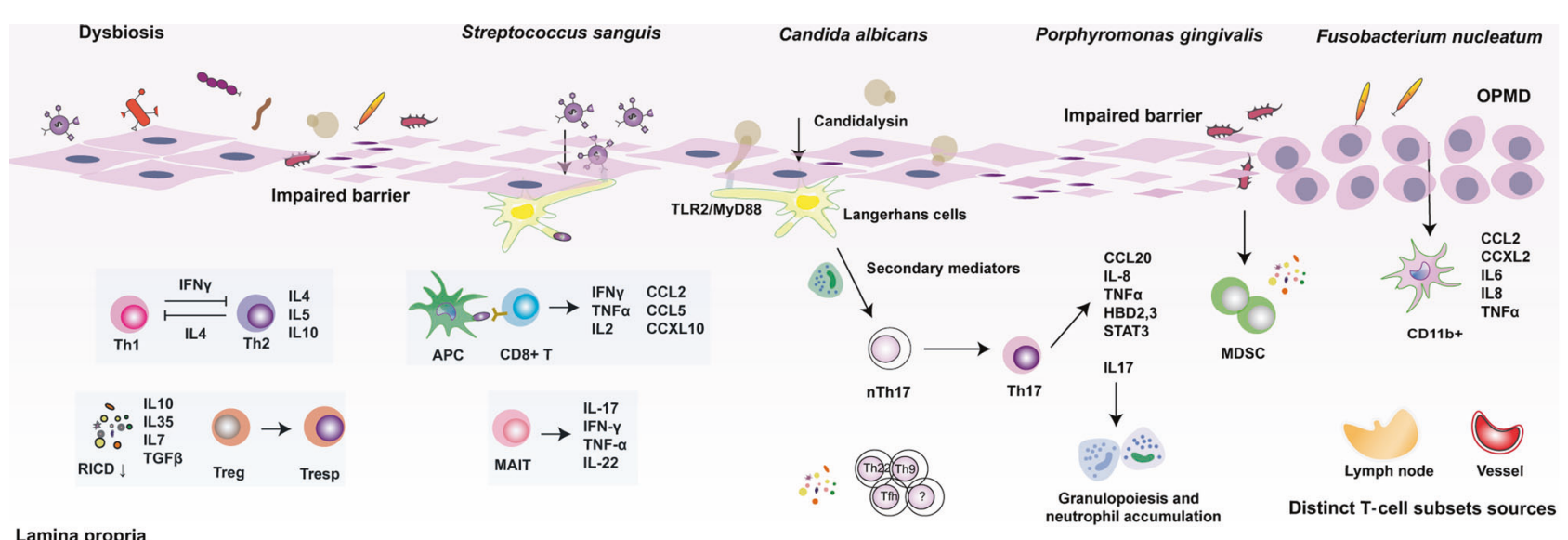

Fig. 2 Crosstalk between the oral dysbiosis and immune cells in oral mucosal diseases (OMDs). $\mathrm{CD} 4^{+} \mathrm{CD} 25^{+}$Foxp $3^{+}$Tregs undergo reduced cell death because they are refractory to TCR restimulation-induced cell death (RICD). The enhanced viability is dependent on increased transforming growth factor- $\beta 1$ (TGF- $\beta 1$ ) signaling that results in upregulation of cellular FADD-like IL- $1 \beta$-converting enzyme (FLICE)inhibitory protein (cFLIP) in Tregs. In this process, Treg cells induce IL-17 cytokines in responding CD4 ${ }^{+}$(Tresp) cells. Streptococcus sanguis (S. sanguis) may initiate a local immune response stimulating Langerhans cells. Th2-type cytokine levels significantly increased in oral lichen planus (OLP) patients. CD8 ${ }^{+} \mathrm{T}$ cell infiltration predicts OLP remission and follows malignant epithelial changes in tissues. MAIT cells rapidly release IL-17, IFN- $\gamma$, TNF- $\alpha$, and IL-22 to help coordinate appropriate immune response. Candida albicans (C. albicans) epitopes can activate STAT3 (necessary for Th17 proliferation and function) through secondary mediators, ensuring initial pattern recognition and providing a cytokine environment to activate Th17 responses dependeding on the TLR2/MyD88 pathway. IL-17 is produced within 1-2 d by $\mathrm{CD}^{+}{ }^{+} \mathrm{CD} 4{ }^{+} \mathrm{CD} 44 \mathrm{hiTCR} \beta^{+} \mathrm{CCR} 6^{+}$natural Th 17 (nTh17) cells and tongue-resident populations of $\gamma \delta$ T cells. IL-17 promotes granulopoiesis and neutrophil accumulation. Porphyromonas gingivalis ( $P$. gingivalis) induces an increase in $C D 11 b^{+}$bone marrow cells and bone marrow-derived inhibitory cells (myeloid-derived suppressor cells, MDSCs) in oral leukoplakia (OLK). CXCL2, CCL2, IL-6, and IL-8 may be potential candidate genes that facilitate MDSC recruitment. OMPD, oral potentially malignant disorders.

recovery from infection (Fig. 2). ${ }^{31}$ However, the signals modulating these subsets that are unique to each mucosal environment in different epithelial cell contexts are unclear. Future studies should concentrate more on directly comparing the similarities and differences in the development and functions of these subsets at locations other than the oral mucosa.

\section{C. albicans-epithelium interaction during oral candidiasis}

The oral epithelium, which is the first barrier against $C$. albicans invasion, is also directly destroyed during $C$. albicans infection. Once attached to host surfaces, $C$. albicans can switch from the yeast to filamentous form, which may facilitate epithelial penetration and the secretion of a cytolytic peptide toxin called candidalysin. ${ }^{53-55}$ Generally, the yeast state of $C$. albicans does not cause damage to the oral epithelium, but hyphal cells exhibit directional growth in response to contact with a surface (thigmotropism), allowing fungi to specifically invade intercellular junctions. ${ }^{56}$ Oral epithelial cells (OECs) are the first to sense the $C$. albicans transition from a benign yeast morphotype to a damaging, invasive hyphal state. ${ }^{29}$ This early recognition is partially mediated by epidermal growth factor receptor (EGFR) family receptors and involves sensing the oral tissue damage induced by candidalysin. ${ }^{40}$ In OECs, candidalysin induces calcium ion influx and lactate dehydrogenase (LDH) release, which are characteristics of cell damage and membrane destabilization. ${ }^{25}$ In addition, $C$. albicans hemolysin can destroy OECs, activate MAPK signal transduction induced by EGFR, trigger the production of inflammatory cytokines, recruit neutrophils, and induce OECs to secrete EGFR ligands and calcium influx. ${ }^{40,57}$ In addition to inducing epithelial damage during the process of entering endothelial cells or the submucosa, C. albicans can invade cells by a passive fungus-induced but host cell-driven process in which lytic enzymes and invasins expressed on hyphae bind and degrade E-cadherin and other interepithelial cell junctional proteins, enabling the organism to penetrate OECs (Fig. 3). ${ }^{54}$ Together, the mechanisms that may tip the balance between disease and restoration of health in the context of $C$. albicans infection are intriguing and likely complex and multifactorial in nature. A greater understanding in this area could undoubtedly provide new avenues to improve current therapies against this pathogenic fungus.

\section{MICROBIAL MODULATION IN ORAL LICHEN PLANUS}

OLP is among the most common chronic inflammatory OMDs and has been estimated to affect $1-2 \%$ of the population. ${ }^{2}$ In clinical settings, OLP is classified into three subtypes (reticular, atrophic, and ulcerative) and affects the buccal mucosa in most cases. The gingiva, tongue, and lips may also be affected. ${ }^{58}$ Various factors have been considered potential causes of OLP, including infection, immunity, genetic factors, stress, and trauma. ${ }^{26}$ However, the precise roles of these factors have been debated. Over the last decade, microbial infection has received increasing attention in the context of OLP pathogenesis. Given the increasing evidence suggesting that bacteria are abundant throughout the epithelium and the lamina propria in OLP and are positively correlated with the levels of infiltrated $\mathrm{CD}^{+}, \mathrm{CD}^{+}$, and $\mathrm{CD} 8^{+}$ cells, ${ }^{59}$ we suggest that the oral microbiota may be a potential trigger factor of OLP onset. ${ }^{26} \mathrm{~A}$ recent description and characterization of the oral microbiota in OLP, which is described below, has facilitated striking observations in a significant proportion of patients with OLP compared to healthy controls. ${ }^{14,60}$ However, the disease onset is unstudied as only cross-sectional human studies currently exist. The longitudinal monitoring of patient oral microbiomes is necessary for evaluating their causative contribution to OLP.

Microbial dysbiosis is diverse in different types of OLP

With the rise of microbiome sequencing in recent years, studies have increasingly demonstrated that microbial dysbiosis may play a causing role in OLP development and that oral microflora changes are significant and unique in this disease. ${ }^{26,61,62}$ To date, the biodiversity of the mycobiome, which is an important component of the oral microbial community, and the roles of bacterial-fungal-virus interactions in OLP pathogenesis remain largely uncharacterized. 


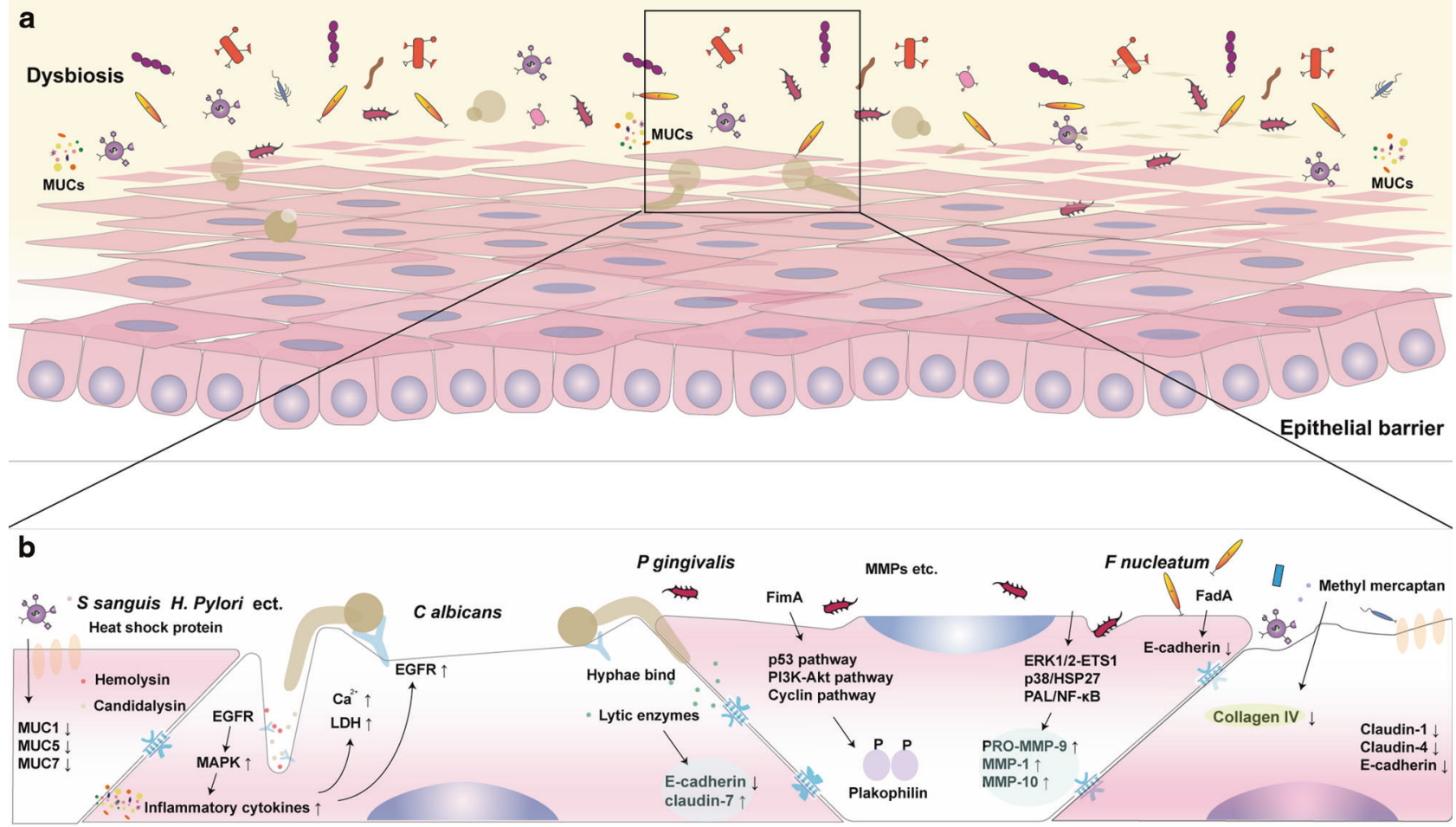

Oral epithelial cells

C

Inflammatory cytokines $\uparrow$

Cell damage Apoptosis $\uparrow$

Membrane destabilization

DNA breakage
Mucosal permeability $\uparrow$
Inflammatory cytokines $\uparrow$

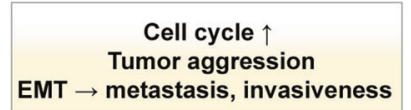

Fig. 3 Crosstalk between the oral dysbiosis and epithelial barrier in oral mucosal diseases (OMDs). a Oral dysbiosis may affect epithelial barrier function followed by the occurrence and progression of OMDs. b, c The $65 \mathrm{kDa}$ heat shock protein produced by Streptococcus sanguis (S. sanguis) causes the production of anti-oral mucosa antibodies and promotes the occurrence of oral ulcers. Mucin proteins, including transmembrane mucin 1 (MUC1) and salivary mucins MUC5B and MUC7, have been shown to play a role in the formation of protective mucosal pellicle, which serves as the first line of defense between the oral epithelium and pathogens within the oral cavity. In oral epithelial cells (OECs), Candida albicans (C. albicans) candidalysin induces calcium ion influx and lactate dehydrogenase (LDH) release. C. albicans hemolysin can destroy OECs; activate MAPK signal transduction induced by EGFR; trigger the production of inflammatory cytokines, including IL-1; as well as induce OECs to secrete EGFR ligands and calcium influx. In addition to inducing epithelial damage in the process of entering endothelial cells or the submucosa, C. albicans can also invade cells by a passive fungus-induced but host cell-driven process whereby lytic enzymes and invasins expressed on hyphae bind to and degrade E-cadherin and other interepithelial cell junctional proteins, enabling the organism to penetrate OECs. Decreased expression of claudin-1, claudin-4, and E-cadherin in oral lichen planus (OLP) may lead to the disorder of epithelial tight junction, cell-cell junction and epithelial permeability, which may lead to the pathogenesis of OLP. Porphyromonas gingivalis ( $P$. gingivalis) has been shown to induce the EMT and can accelerate the cell cycle by affecting the p53, PI3K and cyclin pathways, primarily through its FimA adhesin molecule. The bacterium also downgrades the activity of plakophilin and promotes metastatic change. $P$. gingivalis increases tumor aggression by inducing the excessive expression of matrix metalloproteinase-9 (PRO-MMP-9), MMP-1, and MMP-10 by activating the ERK1/2-ETS1, p38/HSP27, and PAL/NF-KB pathways, thereby worsening the EMT and increasing the invasiveness of tumors. An adhesin of Fusobacterium nucleatum ( $F$. nucleatum), i.e., FadA, can bind E-cadherin on epithelial cells, deactivating it to promote mucosal permeability. In addition, a metabolite of microorganisms, i.e., methyl mercaptan, has been implicated in collagen breakdown, including type 4.

Microorganisms also vary across different OLP types with overall lower levels of fungi and higher levels of bacteria. ${ }^{14,62}$ Recent studies have shown that the abundances of Porphyromonas, Fusobacterium, Leptotrichia, Lautropia, and Solobacterium are significantly increased, while the abundances of Haemophilus, Corynebacterium, Cellulosimicrobium, Campylobacter, and Streptococcus are decreased in OLP. ${ }^{60,63} \mathrm{Li}$ et al. observed that the bacterial community in OLP patient saliva was characterized by greater variety and less bacterial specificity, comprising only Porphyromonas and Solobacterium, which exhibited significantly higher abundances than those in healthy control saliva. ${ }^{62}$ In addition, a decrease in Streptococcus abundance and enrichment of gingivitis/periodontitis-associated bacteria were observed in OLP lesions in another study. ${ }^{59}$ These findings implicate a link between oral bacterial dysbiosis and OLP. Surprisingly, bacteria were even detected within infiltrated T cells. ${ }^{59}$ The presence of bacteria within tissue provides insight into the pathogenetic mechanism of OLP. For example, Porphyromonas gingivalis ( $P$. gingivalis) lipopolysaccharide induces the overproduction of $C C$ chemokine ligand 2 (CCL2) via toll-like receptor-4 (TLR-4)/NF-KB signaling in OLP, which may sustain or exacerbate the chronic inflammation of OLP. ${ }^{64}$

Fungal disorders are related to the aggravation of OLP. ${ }^{62}$ Candida was detected in $37 \%$ of patients, ${ }^{65}$ while Aspergillus was 
identified as an "OLP-associated" fungus because of its higher frequency detection in patients than healthy controls. Compared to healthy controls, significantly higher abundances of Candida and Aspergillus were observed in patients with erosive OLP, and higher abundances of Alternaria and Sclerotiniaceae_unidentified were observed in patients with reticular OLP. ${ }^{62}$ Several keystone fungal genera (Bovista, Erysiphe, Psathyrella, etc.) demonstrated significant correlations with clinical scores and IL-17 levels. ${ }^{66}$ Fungal dysbiosis could alter the salivary bacteriome or may reflect a direct effect of host immunity, which participates in OLP pathogenesis. A recent study has demonstrated negative associations between specific fungal and bacterial taxa identified in healthy controls, which were diminished in reticular OLP patients and even became positive in erosive OLP patients. Moreover, the oral cavities of OLP patients were colonized by a dysbiotic oral flora with lower ecological network complexity and decreased fungalFirmicutes and increased fungal-Bacteroidetes subnetworks. ${ }^{62}$

Potential function of lymphocytic infiltration in defending against dysbiosis in oral lichen planus

The typical histopathological feature of OLP is band-like lymphocytic infiltration in the lamina propria. ${ }^{58}$ Recent research has shown that $\mathrm{CD}^{+}{ }^{+} \mathrm{T}$ lymphocytes are usually distributed in the intraepithelial region or the area from the basal layer to the upper half of the epithelium. This phenomenon occurs because $\mathrm{CD} 8^{+}$ T cells follow malignant epithelial changes in tissues. ${ }^{67}$ In addition, $\mathrm{CD}^{+}{ }^{+}$-cell infiltration predicts OLP remission (Fig. 2). High-grade $\mathrm{CD}^{+}{ }^{+} \mathrm{T}$-cell infiltration is related to a high remission rate. $\mathrm{A}$ diagnostic cutoff value of $\mathrm{CD}^{+}{ }^{+} \mathrm{T}$ cells has been established to predict remission. The present classification of OLP by intraepithelial $\mathrm{CD}^{+}$lymphocyte infiltration may also be helpful for etiological analyses. Remission lesions are presumably caused by transient inducers, such as viral infections. ${ }^{68}$ In addition, most $\mathrm{CD}^{+}$cells are localized in deeper connective tissue. ${ }^{61}$ Recent studies have found that the IL-17 mRNA levels are elevated in local OLP lesions and greatly increase in the serum of female erosive OLP patients, indicating that the Th17 subset may be involved in OLP disease immunopathogenesis. ${ }^{69}$ The salivary concentrations of IL-17 in subjects with erosive OLP are significantly higher than those in subjects with reticular OLP and healthy controls. Moreover, significant positive correlations are observed between the salivary IL-17 concentrations and disease clinical scores. These findings suggest that the salivary bacterial diversity and complexity in subjects with OLP are significantly lower than those in healthy controls and that the shifted community composition is closely related to the immune cytokine IL-17. ${ }^{30}$ Another study show that the Th2-type cytokine levels significantly increase in OLP patients, even in peripheral blood and saliva. In contrast, studies conclude that Th17-associated cytokines may be responsible for more evident oral mucosal damage in erosive OLP, while the elevated Th2 cell levels can explain the less evident epithelial tissue damage involved in reticular OLP (Fig. 2). ${ }^{70}$

Regulation of oral pathogens in the epithelial barrier in oral lichen planus

The abnormal features of the OLP epithelium, such as atrophy, hyperkeratosis, acanthosis, and liquefaction of the basal layer, suggest barrier dysfunction. ${ }^{71}$ Diverse pathogens can modulate the physical barrier function of the epithelia to facilitate infection. ${ }^{72}$ Danielsson et al. analyzed the transcriptome of the OLP epithelium and found that the differentially expressed genes were involved in epithelial differentiation and development. ${ }^{72}$ Based on evidence suggesting that bacteria are abundant throughout the epithelium, Choi demonstrated that certain oral bacteria damage the epithelial physical barrier and are internalized into OECs. ${ }^{59}$ It has been found that the epithelial barrier in OLP tissue was destroyed because many bacterial signals could be detected in both the epithelial basal layer and the lamina propria. ${ }^{59}$ Cadherin-1 (E-cadherin) is expressed in epithelial cells and is an important cell adhesion molecule. Cadherin-1 plays a role in cell growth, differentiation, migration and polarity. ${ }^{73}$ The loss of E-cadherin is a hallmark of the epithelial-to-mesenchymal transition (EMT). ${ }^{74}$ There are differing findings concerning Ecadherin expression in OLP. Du and Li reported that abnormal positive E-cadherin expression occurs in OLP, ${ }^{75}$ whereas Sridevi et al. noted decreased E-cadherin expression in OLP. ${ }^{73}$ Hämäläinen et al. studied the expression of known EMT markers in OLP and reported that the decreased expression of claudin-1, claudin-4 and E-cadherin in OLP may lead to disorder of epithelial tight junctions (TJs), cell-cell junctions and epithelial permeability, which may promote OLP pathogenesis (Fig. 3). ${ }^{76}$ However, the direct relationship between this epithelial disorder and microorganisms has not been clearly discussed.

\section{IMPACT OF THE ORAL MICROBIOTA ON RECURRENT APHTHOUS ULCER}

RAU, which is also known as recurrent aphthous stomatitis (RAS), is the most common lesion observed by clinicians who manage oral ulcerative disease and affects up to $5-20 \%$ of the population. ${ }^{77,78}$ The etiology is unknown, but several factors have been implicated, all of which influence the composition of the oral mucosa and saliva-resident microbiota, which, in turn, modulates immunity and thereby affects disease progression ${ }^{15,79}$

Microbial dysbiosis indicates disease progression in recurrent aphthous ulcer

The cause of RAU is idiopathic and multifactorial. However, aphthous ulcers are more prevalent in individuals with poor oral hygiene practices, ${ }^{80}$ suggesting that oral microbial disorders may participate in the regulation of immune dysfunction and eventually lead to intraepithelial blisters and barrier damage. Interestingly, recent studies have provided compelling evidence suggesting that the oral microbiota can indicate different disease progression in RAU patients. For example, increased abundances of Porphyromonadaceae and Veillonellaceae species were observed only in ulcerated sites, suggesting that these changes are unlikely involved in the initiation of RAS. Moreover, a coinciding decrease in the abundances of Streptococcaceae species was also observed only in active ulcers. ${ }^{15}$ Some bacteria, such as the class Clostridia and genera Lachnoanaerobaculum, Cardiobacterium, Leptotrichia, and Fusobacterium, were also reported to be related to active ulcers. Furthermore, active ulcers were dominated by Malassezia, which was negatively correlated with Streptococcus and Haemophilus and positively correlated with Porphyromonas species. ${ }^{17,81}$ This study demonstrated that the composition of the bacteria and fungi colonizing the healthy oral mucosa was changed in active RAU lesions and that this alteration persists to some extent even after the ulcer is healed. Specifically, Selenomonas was tightly related to RAU recovery. ${ }^{17}$ Compared to that in saliva from healthy controls, the bacterial diversity of saliva in RAU patients was significantly reduced and suggestively correlated with disease activity as evaluated by a quantitative list of disease scores. ${ }^{15}$ No individual pathogens had been conclusively shown to be correlative agents of RAS until Kim et al. acknowledged that decreased Streptococcus salivarius (S. salivarius) and increased Acinetobacter johnsonii (A. johnsonii) abundances in the mucosa were associated with RAU risk. A dysbiosis index developed using the relative abundances of $A$. johnsonii and $S$. salivarius and regression coefficients correctly predicted $83 \%$ of the total cases in the absence or presence of RAU. ${ }^{82}$ Another recent study also has suggested that RAU occurrence is significantly associated with an increase in Escherichia coli and Alloprevotella and a decrease in Streptococcus abundances. ${ }^{79}$

As discussed above, the identification of protective symbionts and pathobionts that contribute to disease progression could be 
crucial for the development of effective RAU treatment. These studies still do not provide clear data concerning causality. A comparison of mucosal microbiomes from patients associated with similar immune pathogenesis but different etiologies may provide data related to causality.

\section{Microbiome-infected cytotoxic T lymphocytes cause recurrent} aphthous ulcer

Increasing evidence links local immune dysfunction to RAU, although the specific defect remains unknown. Over the past 30 years, research has suggested that a relationship exists among RAU, lymphocytotoxicity, antibody-dependent cell-mediated cytotoxicity, defects in lymphocyte cell subpopulations, and an alteration in the CD4 to CD8 lymphocyte ratio. ${ }^{83}$ RAU is initially and primarily the result of $\mathrm{T}$ cell-mediated immune dysfunction but may also involve neutrophil and mast cell-mediated destruction of the mucosal epithelium. ${ }^{81}$ Lesions can exhibit alterations in several intercellular mediators, such as elevations in the levels of interferon gamma (IFN- $\gamma$ ), tumor necrosis factor-alpha (TNFa), IL-2, IL-4, IL-5, and various adhesion molecules involve in cell communication and epithelial integrity. However, a reverse relationship exists between bacterial diversity and some inflammatory cytokines in saliva, such as IFN- - , IL-4, and IL-17. ${ }^{3}$ Finally, this inflammatory process results in a pseudomembrane containing fibrinous exudate, bacteria, inflammatory cells, and necrotic mucosal cells.

Some scientists have suggested that the lysis of mucosal epithelial cells caused by microbiome-infected cytotoxic $T$ lymphocytes (CTLs) may be the reason for the occurrence of ulcers. ${ }^{84,85}$ Once ulceration occurs, bacteria, such as Streptococcus, can contact ulcerative oral lesions through mucous membrane breaks. Streptococcus and its associated antigens penetrate the ulcerative oral mucosa to further trigger specific immune responses, resulting in CTL infiltration into the epithelial and inherent layers of the oral mucosa. ${ }^{85}$ For example, Stehlikova et al. suggested that a high load of Streptococcus sanguis (S. sanguis)like microorganisms may initiate a local immune response stimulating Langerhans cells (Fig. 2) and activating a crossreacting autoimmune response to homologous peptides within epithelial heat shock proteins. This process can initiate immunopathological changes that lead to RAU. These newly generated CTLs destroy adjacent epithelial cells and further lead to the formation of oral ulcers during the late-middle and late stages of RAU deterioration. ${ }^{17}$ During the healing phase, the T-cell proliferation response decreases to normal levels, giving the OECs at the edge of the ulcer an opportunity to proliferate and bind the mucous membrane, eventually leading to healing, which has also been observed in RAU patients with acute exacerbation; the percentages of $\mathrm{CD}^{+}, \mathrm{CD}^{+}$, and $\mathrm{CD} 8 \mathrm{IL}-2 \mathrm{R}^{+}$cells in peripheral blood is increased, and this percentage returns to normal levels after acute exacerbation. ${ }^{86}$ The positive effect of antimicrobial therapy on RAU suggests that the oral microbiota is potentially involved in the etiology and pathogenesis of RAU, although its complex pathophysiology cannot be attributed to a single pathogen.

Microbiome-dependent regulation in the epithelium in recurrent aphthous ulcer

Mucosal injury due to a similar barrier function deficiency has been shown in other parts of the body, such as the eyes, skin, airways, and intestines. Inflammatory bowel diseases are prototypes of this type of injury, and these diseases have been well studied. ${ }^{23,32,87}$ Although the interaction between the commensal flora and the covering layer of the epithelium may be quite similar in the oral mucosa and intestine, there are distinctions.

Recent evidence suggests that the damage to epithelial integrity resulting from microorganisms also plays an important role in RAU formation. ${ }^{88}$ It is known that RAU occurs on only nonkeratinized oral mucosa but not on keratinized oral mucosa, which may be related to the significant difference in the composition of the microbiome between keratinized mucosa and nonkeratinized mucosa. ${ }^{77,89,90}$ Moreover, RAU is relatively rare in smokers, while the oral microbiomes in smokers and nonsmokers are obviously dissimilar. Studies have suggested that smoking may improve some mechanical properties of the oral epithelium by increasing its thickness and promoting keratosis, which is consistent with the rare occurrence of RAU in the keratinized oral mucosae. ${ }^{91,92}$ Interestingly, A. johnsonii substantially inhibits the proliferation of gingival epithelial cells and shows greater cytotoxicity against gingival epithelial cells than $S$. salivarius. $^{82}$ In addition, the metabolites produced by microbes damage the oral epithelium structure and may cause RAU development. Researchers have indicated that the $65 \mathrm{kDa}$ heat shock protein produced by the oral symbiotes $S$. sanguis and Mycobacterium can cross-react with peptides in OECs, causing the production of anti-oral mucosa antibodies and promoting the occurrence of oral ulcers (Fig. 3). ${ }^{17}$ More recently, some scientists emphasize the role of the mucin proteins in the pathophysiology of RAU. ${ }^{93}$ Mucin proteins, including transmembrane mucin 1 (MUC1) and salivary mucins MUC5B and MUC7, have been shown to play a role in the formation of protective mucosal pellicle, which serves as the first line of defense between the oral epithelium and pathogens within the oral cavity. MUC1 limits the binding of $H$. Pylori to gastric epithelial cells in mice, and adenoviral penetrance into the epithelium in airways is increased in specimens lacking MUC1. ${ }^{94}$ Interestingly, a study found that the salivary MUC1 concentration is decreased in patients with stress, ${ }^{95}$ which is considered a predisposing factor of RAU. MUC7 has been shown to have an altered structure, i.e., a loss of terminal oligosaccharides, in patients with RAS; additionally, due to the loss of sialic acid in its terminal end, MUC7 loses its ability to adhere to Streptococci. ${ }^{96}$ The thickness and composition of the mucin proteins differs according to their localization in the oral cavity and the level of keratinization, ${ }^{97}$ explaining why RAS mainly develops in the nonkeratinized epithelium. ${ }^{98}$ How do microbial pathogens penetrate the oral mucosal barrier? Parssinen et al. hypothesized that deficiencies in salivary mucin (MUC5B and MUC7) formation or a decrease in mucosal transmembrane mucin (MUC1) due to proteolytic bacteria could be a predisposing factor of RAU (Fig. 3). ${ }^{93}$ However, the promising topic of the causative mechanisms between oral microbes and the epithelium participating in RAU development is still poorly discussed and requires further investigation.

\section{MICROBIAL SIGNALS IN ORAL LEUKOPLAKIA AND ORAL SQUAMOUS CELL CARCINOMA}

OLK is defined as a predominantly white lesion of the oral mucosa that cannot be wiped off the mucosa or ascribed to any specific disease process. ${ }^{7,9}$ OLK is a pathological diagnosis. The histopathologic features of the epithelium may include hyperkeratosis, atrophy, and hyperplasia with or without dysplasia., ${ }^{5,-9}$ Usually, OLK belongs to the white lesions of the oral mucosa or oral premalignancy in different classifications of OMD., ${ }^{5-9}$ OLK is among the most common oral potentially malignant disorders (OPMDs), and its rate of malignant transformation ranges from $1-20 \%$ depending on the population and the length of followup. ${ }^{4,99}$ OSCC represents the most common malignant neoplasm of the oral cavity and comprises $80-90 \%$ of head and neck cancers. Over the past two decades, the 5-year survival rate has remained at $\sim 50 \%$ due to its initially asymptomatic nature, leading to advanced stage diagnosis with few therapeutic options. ${ }^{100}$ Although smoking, alcohol, and betel nut use are clearly associated with OLK development, the factors driving the malignant transformation of these lesions are poorly understood, and it is difficult to accurately predict whether OLK will resolve, 
persist or progress to OSCC. ${ }^{101}$ The specific microbial enrichment and its metabolites associated with potentially malignant OLK could be used as novel biomarkers of malignant transformation. Various hypotheses have been hyd to link microorganisms and their products with OLK and OSCC. ${ }^{102-105}$

Oral microbiota and metabolites: potential biomarkers of malignant transformation

Microbiome studies have been carried out to identify changes in the microbiota and metabolites in OLK and OSCC patients with the hope of identifying biomarkers of malignant transformation. ${ }^{18,19,106}$

Although colonization with C. albicans is common, OLK exhibits an increased abundance of Fusobacteria and reduced levels of Firmicutes. The bacterial colonization patterns in OLK are highly variable, and five distinct bacterial clusters have been discerned. These clusters exhibit the cooccurrence of Fusobacterium, Leptotrichia, and Campylobacter species, which is strikingly similar to the microbial cooccurrence patterns observed in colorectal cancers. ${ }^{107-109}$ An increased abundance of the acetaldehydogenic microorganism Rothia mucilaginosa ( $R$. mucilaginosa) is also apparent in OLK at lingual sites. Severe dysplasia is associated with elevated levels of Leptotrichia spp. and Campylobacter concisus. ${ }^{102}$ Further study shows that $R$. mucilaginosa can generate acetaldehyde $(\mathrm{ACH})$ from ethanol in vitro at levels inducing oxidative stress, which may play a role in the development of OLK and/or the malignant transformation of OLK to OSCC. ${ }^{110}$ Researchers have indicated that the relative abundances of the phylum Bacteroidetes and genera Streptococcus and Solobacterium are significantly higher in the OSCC group than the OLK group; thus, a shift in the Streptococcus and Solobacterium levels may be considered a novel clinical indicator of potential malignancy in a precancerous lesion. ${ }^{106}$ Another study discussed the comprehensive profile of the oral microbiome during cancer progression from the early stage to the late stage and found that the populations dynamically changed with cancer progression from stage 1 to stage 4 . The oral microbiota communities in the stage 4 patients showed significantly higher complexity than those from the healthy controls. At the genus level, the abundance of Fusobacterium increased, while the numbers of Streptococcus, Haemophilus, Porphyromonas, and Actinomyces decreased with cancer progression. Fusobacterium periodonticum ( $F$. periodonticum), Parvimonas micra, Streptococcus constellatus, Haemophilus influenzae, and Filifactor alocis were associated with OSCC and progressively increased in abundance from stage 1 to stage 4 . The abundances of Streptococcus mitis (S. mitis), Haemophilus parainfluenzae, and Porphyromonas pasteri ( $P$. pasteri) were inversely associated with OSCC progression. A bacterial marker panel comprising three bacteria (upregulated $F$. periodonticum and downregulated S. mitis and $P$. pasteri) had an area under the curve (AUC) of $0.956(95 \% \mathrm{Cl}$ $=0.925-0.986)$ in discriminating OSCC stage 4 patients from the healthy controls. ${ }^{18} P$. gingivalis has been identified as an independent and significant risk factor in cancer-related deaths in the oral cavity and throughout the remaining oral digestive tract. ${ }^{111}$ A cohort study showed that the location of $P$. gingivalis in tumor tissue was associated with poor survival in OSCC patients. ${ }^{112}$ In vivo, $P$. gingivalis infection increases the number and size of oral lesions and promots tumor progression in 4nitronolyn-1 oxide (4NQO)-induced cancer mouse models by invading oral lesions. ${ }^{112}$

In addition, several damaging metabolic end products, such as volatile sulfur compounds, organic acids, and aldehydes, and nitrosatable compounds are reported to be associated with the development of oral cancer. ${ }^{27}$ Many oral microbiota, including the Streptococcus species (S. gordonii, S. mitis, S. oralis, S. salivarius, and S. sanguinis), Rothia species, $P$. gingivalis and C. albicans, ${ }^{113}$ produce $\mathrm{ACH}$, which has the ability to exert DNA damage and cause excess proliferation in the epithelium. Bacteria and fungi also can catalyze nitrosatable compounds to form N-nitroso compounds through nitridation pathways. ${ }^{114}$ Kakabadze hypothesized that Pseudomona aeruginosa ( $P$. aeruginosa) increases the concentration of NO by converting salivary nitrite to nitric oxide, thereby contributing to NO-related carcinogenesis. ${ }^{115}$ Volatile sulfur compounds, predominantly including hydrogen sulfide and methyl mercaptan, produced by periodontal pathogens, such as $P$. gingivalis, Prevotella intermedia and Fusobacterium nucleatum ( $F$. nucleatum), have been implicated in oxidative stress and DNA damage in oral cells. Host proteins may also be metabolized or fermented into sulfides and nitrosamines by Firmicutes and Bacteroides, potentiating cell mutations. ${ }^{116}$ In contrast, hydrogen peroxide may be involved in oral cancer preventative pathways, and evidence suggests that oral bacterial hydrogen peroxide can suppress the significantly higher expression of NLRP3 inflammasomes in OSCC cells, ${ }^{117}$ which is associated with increased tumor sizes and lymph node metastasis. ${ }^{118}$ Terpenoids and polyketides are secondary metabolites produced by certain microorganisms and possess potent pharmaceutical activities against cancer. ${ }^{119,120}$ Recently, a study suggests that compared with the controls, microbial pathway modules associated with the metabolism of terpenoids and polyketides, including the biosynthesis of siderophore group nonribosomal peptides, monoterpenoid biosynthesis, and biosynthesis of 12-, 14- and 16-membered macrolides, are less abundant in oral tumor lesions. ${ }^{28}$

In conclusion, these findings reveal that the oral microbiota community dynamically changes and potentially induces oral lesion progression. These findings highlight a novel aspect of OLK and OSCC etiology and the functional role of the oral microbiome in formulating a tumor microenvironment via the attenuated biosynthesis of secondary metabolites with cancer-promoting or anti-cancer effects. The oral microbiota and its metabolites are compositionally and functionally associated with the development of oral cancer. Our review suggests that specific oral microbes and their metabolites could be potential biomarkers for the early diagnosis and prognosis monitoring of this deadly malignancy.

Specific pathogens orchestrate the immune microenvironment of oral lesions

Over the past decade, there have been advances in the microbiota-immune pathological aspects of OLK and OSCC. The early stage of the tumor carcinogenesis process is related to immune response changes in cytokine levels, immune cell density, and immune cell function. ${ }^{121}$ How do pathogens interact with the immune response beyond inducing inflammation? Tregs play a key immune regulatory role during infection and are essential for ensuring the effective prevention of pathogens and control of infection-related immunopathology, which has been widely studied in gastrointestinal homeostasis and diseases. ${ }^{34,122}$ Studies have demonstrated that Treg and Th17 cell functions are associated with oral Candida, which is the predominant fungal species associated with $\mathrm{OLK}^{34}$ In vivo experiments illustrated that a mouse Candida infection model had an increased proportion of Foxp $3^{+}$Tregs in the oral mucosa and cervical lymph nodes, which depended on the TLR2/MyD88 pathway (Fig. 2). Reducing the levels of these cells coincided with an increase in fungal burdens in the histopathological examination and oral mucus, ${ }^{31,51}$ suggesting that symbiotic bacteria are important for controlling the mucous membrane immunity mediated by Foxp3 cells and Th17 cells (Fig. 2). Compared to healthy individuals, the levels of IL-6, IL-8, and TNFa in OPMD patients are significantly increased, ${ }^{16}$ which may indicate a transition from a benign to malignant state. ${ }^{123}$ Studies have shown that $P$. gingivalis induced an increase in $\mathrm{CD}_{11} \mathrm{~b}^{+}$bone marrow cells and bone marrow-derived inhibitory cells (myeloid-derived suppressor cells, MDSCs) in OLK (Fig. 2). In vitro observations showed that MDSCs accumulated 
when human-derived dysplastic oral keratinocytes were exposed to $P$. gingivalis and that $C X C L 2, C C L 2, I L-6$, and IL-8 may be potential candidate genes that facilitate MDSC recruitment (Fig. 2). ${ }^{112} P$. gingivalis can help OSCC cells bypass the immune system by activating PD-L1 to bind its receptor, i.e., PD-1, thereby mediating profound T-cell inhibition, ${ }^{124}$ and can induce the expression of the $\mathrm{B} 7-\mathrm{H} 1$ and $\mathrm{B} 7-\mathrm{DC}$ receptors in OSCC cells, leading to apoptosis in activated $\mathrm{T}$ cells. ${ }^{125}$ Fusobacterial proteins, such as Fap 2, can block and downgrade NK and T cell activity by the binding of Fap 2 with the inhibitory $T$ cell immunoreceptor. ${ }^{126,127}$ Thus, these three species, C. Candida, $P$. gingivalis and $F$. nucleatum, have considerable virulence characteristics that allow them to be significantly involved in OSCC progression.

In conclusion, the immune system plays an important role in the microenvironment of preneoplastic and neoplastic lesions; knowledge regarding the role of the oral microbiota in controlling the local aggressiveness, growth, and diffusion of cancer cells still requires further investigation.

Interaction between the oral microbiota and epithelial malignancy The oral mucosa comprises the epithelium and stroma, providing the initial physical defense against infection. Epithelial mesenchymal interactions are essential for cell growth, differentiation, and tumorigenesis. ${ }^{128}$ The EMT is critical for the conversion of OECs into carcinoma cells during carcinogenesis. ${ }^{129}$ Several specific pathogens have been reported to potentially trigger the EMT through different mechanisms. $P$. gingivalis has been shown to induce the EMT and can accelerate the cell cycle by affecting the p53, PI3K, and cyclin pathways, ${ }^{130}$ primarily through its FimA adhesin molecule. $^{131}$ The bacterium also downgrades the activity of plakophilin, which is a key molecule in epithelial cells, and, thus, can promote metastatic change. ${ }^{132}$ It is currently believed that $P$. gingivalis increases tumor aggression by inducing the excessive expression of matrix metalloproteinase-9 (PRO-MMP-9), MMP-1, and MMP-10 by activating the ERK1/2-ETS1, p38/HSP27, and PAL/ NF-KB pathways, thereby worsening the EMT and increasing the invasiveness of tumors (Fig. 3). ${ }^{133}$ In addition, an adhesin of $F$. nucleatum, i.e., FadA, can bind E-cadherin on epithelial cells, deactivating it to promote mucosal permeability (Fig. 3). P. aeruginosa is a rare species isolated from OSCC but has been implicated in carcinogenesis due to its ability to cause DNA breakage in epithelial cells ${ }^{134}$ and promote invasion and metastatic change. In addition, a metabolite of microorganisms, i.e., methyl mercaptan, has been implicated in collagen breakdown, including type 4, and, thus, may play a role in OSCC invasion across the basement membrane (Fig. 3). ${ }^{135}$

Epithelial barrier disfunction facilitates oral pathogen infiltration. Cheng et al. observed that epithelial barrier disorder and the altered biological characteristics of the adjacent stroma (fibroblasts) were conducive to C. albicans infection in OLK, which, in turn, promoted disease progression. ${ }^{136}$ Intercellular junctions are important structures for the physiological functions of cells. ${ }^{137}$ TJs play a main role in signaling cascades that control cell growth and differentiation. ${ }^{138}$ Abnormalities in TJ permeability allow increased pathogen infections that may promote tumor growth. Changes in TJs have been noted as an early event in tumor metastasis, ${ }^{139}$ especially the downregulation or upregulation of claudins and occludin (Fig. 3). ${ }^{140}$ Phattarataratip et al. showed that there was a tendency toward an association between higher claudin-7 expression and a longer survival time. ${ }^{140}$ However, the influence of the higher claudin-7 expression on pathogen infection has not been well studied. The interaction between microorganisms and the epithelial barrier is well known in the intestinal microecosystem. ${ }^{23}$ How oral microorganisms and their metabolites affect the malignancy and metastasis of OSCC through crosstalk with oral mucosal cells is still rarely reported. There is a great need for additional research in this field.

\section{CONCLUSION AND PERSPECTIVES}

In recent decades, our ability to identify and culture oral microbial residents and decipher their wide range of interactions has been significantly improved. Currently, microbiome research using nextgeneration sequencing has admittedly reached a peak in productivity, aligning with advancing technological trends. To date, most microbiota studies have relied on analyzing microbiota composition via 16S rRNA gene sequencing of salivary or oral mucosa. However, an understanding of how the oral microbiome, which is an ecosystem with diverse interactive activities that participates in the oral mucosal epithelial barrier and dynamic balance of the immune system, requires further exploration. In this narrative literature review, we highlight that oral mucosal microbiology is intensively associated with the pathogenesis of OMD through crosstalk with mucosal immunity and the epithelial barrier. Importantly, we aim to provide a scientific hypothesis guiding further investigations of the pathogenesis mechanisms of OMD. We hypothesize that an altered composition of the mucosal microbiota in the oral cavity, which could stimulate immune imbalance or damage the integrity and tolerance of the epithelial barrier, might be the triggering pathology underlying the occurrence and development of OMD (Box 1).

Despite recent progress in the field, several challenges remain to be addressed and overcome (Box 1). First, the important role of the oral microbiome suggests that we should develop clinical strategies targeting the oral microbiome. However, demonstrating whether oral microbiomes are the cause or consequence of OMD has been proven difficult. Most studies are limited to correlation conclusions and lack causal arguments. Confirmation is essential for the microbiome to become a valid target for interventions, e.g., using persuasive experimental evidence to enact oral microbiota transplantation. Second, the identification of the causal components of complex microbiomes responsible for pathologies is another challenge, although whether their complex pathophysiology could be attributed to a single pathogen remains debatable. Through a comprehensive understanding of these interactions, we could learn how to optimally modulate the oral microbiota to enhance OMD therapies. Third, microbiota-modulated immune cells may differ in each mucosal etiological niche because of the different epithelial cell contexts, which is called site-specific.

\section{Box 1}

Summary of the review.

\section{Hypothesis.}

- Altered composition of mucosal microbiota in the oral cavity, which could stimulate immune imbalance or damage the integrity and tolerance of the epithelial barrier, might be the pathological reason behind the occurrence and development of oral mucosal disease (OMD).

\section{Challenges.}

- It is essential but difficult to demonstrate whether oral microbiomes are the cause or consequence of OMD.

- The identification of the causal components of complex microbiomes responsible for pathologies is another challenge.

- The microbiota-modulated immune cells may differ in each mucosal etiological niche because of the different epithelial cell contexts, which is called site-specific. Future studies could concentrate more on the composition of microbiotamodulated immune cell subsets at different locations of oral mucosa. 
Future studies could concentrate more on the composition of microbiota-modulated immune cell subsets at different locations of the oral mucosa. It is believed that in the future, the continuous expansion and supplementation of mucosal microbiology could greatly enhance our understanding of OMD.

\section{ACKNOWLEDGEMENTS}

This research project was supported by the National Natural Science of Foundation of PR China [Nos. 81772896 and 81972532].

\section{AUTHOR CONTRIBUTIONS}

Z.W. contributed to the conception, design, drafting, and revision of the manuscript. D.L., L.Y. and L.W. reviewed the literature. D.L wrote the manuscript. D.L and H.L. designed and drew the figures. Q.C. and D.L. revised the manuscript.

\section{ADDITIONAL INFORMATION}

Competing interests: The authors declare no competing interests.

Publisher's note Springer Nature remains neutral with regard to jurisdictional claims in published maps and institutional affiliations.

\section{REFERENCES}

1. Vila, T., Sultan, A. S., Montelongo-Jauregui, D. \& Jabra-Rizk, M. A. Oral candidiasis: a disease of opportunity. J. Fungi. 6, 15 (2020)

2. Alrashdan, M. S., Cirillo, N. \& McCullough, M. Oral lichen planus: a literature review and update. Arch. Dermatol Res. 308, 539-551 (2016).

3. Rivera, C. Essentials of recurrent aphthous stomatitis. Biomed. Rep. 11, 47-50 (2019).

4. Ojeda, D., Huber, M. A. \& Kerr, A. R. Oral potentially malignant disorders and oral cavity cancer. Dermatol Clin. 38, 507-521 (2020).

5. Chen, Q., Zeng X. Case Based Oral Mucosal Diseases. 1st edn (Springer Nature Singapore Pte Ltd., Beijing, 2018).

6. Chen, Q. Diseases of Oral Mucosa. 5th edn (People's Medical Publishing House, Beijing, 2020).

7. Greenberg, M., Glick, M., Ship, J. A. Burbet's Oral Medicine. 12th edn (People's Medical Publishing House, Lewiston, NY, 2015).

8. Kahn, M. A. \& Hall, J. M. The ADA Practical Guide to Soft Tissue Oral Disease. 2nd edn (John Wiley \& Sons, Inc. and the ADA, Hoboken, NJ, 2014).

9. Odell, E. W. Cawson's Essentials of Oral Pathology and Oral Medicine. 9th edn (Elsevier, London, 2017).

10. Silverman, S., Eversole, L. Y., Truelove, E. D. Essentials of Oral Medicine. 1st edn (PMPH USA, Ltd, Lewiston, NY, 2001).

11. Petersen, C. \& Round, J. L. Defining dysbiosis and its influence on host immunity and disease. Cell Microbiol. 16, 1024-1033 (2014).

12. Levy, M., Kolodziejczyk, A. A., Thaiss, C. A. \& Elinav, E. Dysbiosis and the immune system. Nat. Rev. Immunol. 17, 219-232 (2017).

13. $\mathrm{Hu}, \mathrm{L}$. J. et al. Characterization of oral candidiasis and the Candida species profile in patients with oral mucosal diseases. Micro. Pathogenesis. 134, 103575 (2019).

14. Yu, F. Y. et al. Dysbiosis of saliva microbiome in patients with oral lichen planus. BMC Microbiol. 20, 75 (2020).

15. Hijazi, K. et al. Mucosal microbiome in patients with recurrent aphthous stomatitis. J. Dent. Res. 94, 87S-94S (2015).

16. Dikova, V. R., Principe, S. \& Bagan, J. V. Salivary inflammatory proteins in patients with oral potentially malignant disorders. J. Clin. Exp. Dent. 11, e659-e664 (2019).

17. Stehlikova, Z. et al. Oral microbiota composition and antimicrobial antibody response in patients with recurrent aphthous stomatitis. Microorganisms 7, 636 (2019).

18. Yang, C. Y. et al. Oral microbiota community dynamics associated with oral squamous cell carcinoma staging. Front Microbiol. 9, 862 (2018).

19. Yang, S. F. et al. Compositional and functional variations of oral microbiota associated with the mutational changes in oral cancer. Oral. Oncol. 77, 1-8 (2018).

20. Verma, D., Garg, P. K. \& Dubey, A. K. Insights into the human oral microbiome. Arch. Microbiol. 200, 525-540 (2018).

21. Blander, J. M., Longman, R. S., Iliev, I. D., Sonnenberg, G. F. \& Artis, D. Regulation of inflammation by microbiota interactions with the host. Nat. Immunol. 18, 851-860 (2017).
22. Wambre, E. \& Jeong, D. Oral tolerance development and maintenance. Immunol. Allergy Clin. N. Am. 38, 27-37 (2018).

23. Amelia, T., Soderholm \& Virginia, A. P. Intestinal epithelial cells: at the interface of the microbiota and mucosal immunity. Immunology 158, 267-280 (2019).

24. Sultan, A. S., Kong, E. F., Rizk, A. M. \& Jabra-Rizk, M. A. The oral microbiome: a lesson in coexistence. PLoS Pathog. 14, e1006719 (2018).

25. Pellon, A., Nasab, S. D. S. \& Moyes, D. L. New insights in Candida albicans innate immunity at the mucosa: toxins, epithelium, metabolism, and beyond. Front Cell Infect. Microbiol. 10, 81 (2020).

26. Baek, K. \& Choi, Y. The microbiology of oral lichen planus: is microbial infection the cause of oral lichen planus? Mol. Oral. Microbiol. 33, 22-28 (2018).

27. Sami, A., Elimairi, I., Stanton, C., Ross, R. P. \& Ryan, C. A. The role of the microbiome in oral squamous cell carcinoma with insight into the microbiometreatment axis. Int. J. Mol. Sci. 21, 8061 (2020).

28. Su, S. C. et al. Oral microbial dysbiosis and its performance in predicting oral cancer. Carcinogenesis 42, 127-135 (2021).

29. Aggor, F. E. Y. et al. Oral epithelial IL-22/STAT3 signaling licenses IL-17-mediated immunity to oral mucosal candidiasis. Sci. Immunol. 5, eaba0570 (2020).

30. Wang, K. et al. Analysis of oral microbial community and Th17-associated cytokines in saliva of patients with oral lichen planus. Microbiol Immunol. 59, 105-113 (2015).

31. Pandiyan, P. et al. $\mathrm{CD} 44^{(+)} \mathrm{CD} 25^{(+)} \mathrm{Foxp}^{(+)}$regulatory $\mathrm{T}$ cells promote Th17 cells in vitro and enhance host resistance in mouse Candida albicans Th17 cell infection model. Immunity 34, 422-434 (2011).

32. Allaire, J. M. et al. The intestinal epithelium: central coordinator of mucosal immunity. Trends Immunol. 40, 174 (2019).

33. Belkaid, Y. \& Hand, T. W. Role of the microbiota in immunity and inflammation. Cell 157, 121-141 (2014).

34. Pandiyan, P. et al. Microbiome dependent regulation of Tregs and Th17 cells in mucosa. Front. Immunol. 10, 426 (2019).

35. Abdulkareem, A. A., Shelton, R. M., Landini, G., Cooper, P. R. \& Milward, M. R. Potential role of periodontal pathogens in compromising epithelial barrier function by inducing epithelial-mesenchymal transition. J. Periodontal Res. 53, 565-574 (2018).

36. Xu, H. \& Dongari-Bagtzoglou, A. Shaping the oral mycobiota: interactions of opportunistic fungi with oral bacteria and the host. Curr. Opin. Microbiol. 26, 65-70 (2015).

37. Bertolini, M. \& Dongari-Bagtzoglou, A. The relationship of Candida albicans with the oral bacterial microbiome in health and disease. Adv. Exp. Med Biol. 1197, 69-78 (2019).

38. Kruger, W., Vielreicher, S., Kapitan, M., Jacobsen, I. D. \& Niemiec, M. J. Fungalbacterial interactions in health and disease. Pathogens 8, 70 (2019).

39. Bertolini, M. et al. Candida albicans induces mucosal bacterial dysbiosis that promotes invasive infection. PLoS Pathog. 15, e1007717 (2019).

40. Naglik, J. R., Konig, A., Hube, B. \& Gaffen, S. L. Candida albicans-epithelial interactions and induction of mucosal innate immunity. Curr. Opin. Microbiol. 40, 104-112 (2017).

41. Meir, J. et al. Identification of Candida albicans regulatory genes governing mucosal infection. Cell Microbiol. 20, e12841 (2018).

42. Conti, H. R. et al. IL-17 receptor signaling in oral epithelial cells is critical for protection against oropharyngeal candidiasis. Cell Host Microbe 20, 606-617 (2016).

43. Conti, H. R. et al. Oral-resident natural Th17 cells and $\gamma \delta T$ cells control opportunistic Candida albicans infections. J. Exp. Med. 211, 2075-2084 (2014).

44. Wilharm, A. et al. Mutual interplay between IL-17-producing $\gamma \delta \mathrm{T}$ cells and microbiota orchestrates oral mucosal homeostasis. Proc. Natl Acad. Sci. USA 116, 2652-2661 (2019).

45. Hernandez-Santos, N. et al. Th17 cells confer long-term adaptive immunity to oral mucosal Candida albicans infections. Mucosal Immunol. 6, 900-910 (2013).

46. Mengesha, B. G. \& Conti, H. R. The role of IL-17 in protection against mucosal Candida infections. J. Fungi. 3, 52 (2017).

47. Zizzo, G. \& Cohen, P. L. IL-17 stimulates differentiation of human sntiinflammatory macrophages and phagocytosis of apoptotic neutrophils in response to IL-10 and glucocorticoids. Immunology 190, 5237-5246 (2013).

48. Rodriguez, M. et al. Fungal pattern receptors down-regulate the inflammatory response by a cross-inhibitory mechanism independent of interleukin-10 production. Immunology 150, 184-198 (2017).

49. Milner, J. D. et al. Impaired $\mathrm{T}(\mathrm{H}) 17$ cell differentiation in subjects with autosomal dominant hyper-IgE syndrome. Nature 452, 773-776 (2008).

50. Whibley, N. et al. Antibody blockade of IL-17 family cytokines in immunity to acute murine oral mucosal candidiasis. J. Leukoc. Biol. 101, 1065-1065 (2017).

51. Bhaskaran, N., Cohen, S., Zhang, Y. F., Weinberg, A. \& Pandiyan, P. TLR-2 signaling promotes IL-17A production in $\mathrm{CD} 4{ }^{(+)} \mathrm{CD} 25^{(+)} \mathrm{Foxp}^{(+)}$regulatory cells during oropharyngeal candidiasis. Pathogens 4, 90-110 (2015). 
52. Bhaskaran, N. et al. Transforming growth factor- $\beta 1$ sustains the survival of Foxp $3^{(+)}$regulatory cells during late phase of oropharyngeal candidiasis infection. Mucosal Immunol. 9, 1015-1026 (2016).

53. Swidergall, M. \& Filler, S. G. Oropharyngeal candidiasis: fungal invasion and epithelial cell responses. PLoS Pathog. 13, e1006056 (2017).

54. Desai, J. V. Candida albicans hyphae: from growth initiation to invasion. J. Fungi. 4, 10 (2018).

55. Moyes, D. L. et al. Candidalysin is a fungal peptide toxin critical for mucosal infection. Nature 532, 64-68 (2016).

56. Hofs, S., Mogavero, S. \& Hube, B. Interaction of Candida albicans with host cells: virulence factors, host defense, escape strategies, and the microbiota. J. Microbiol. 54, 149-169 (2016).

57. Ho, J. et al. Candidalysin activates innate epithelial immune responses via epidermal growth factor receptor. Nat. Commun. 10, 2297 (2019).

58. Gupta, S. \& Jawanda, M. K. Oral lichen planus: an update on etiology, pathogenesis, clinical presentation, diagnosis and management. Indian J. Dermatol. 60, 222-229 (2015).

59. Choi, Y. S. et al. The presence of bacteria within tissue provides insights into the pathogenesis of oral lichen planus. Sci. Rep. 6, 29186 (2016).

60. Du, G. H. et al. Potential association between Fusobacterium nucleatum enrichment on oral mucosal surface and oral lichen planus. Oral. Dis. 26, 122-130 (2020).

61. Wei, W. et al. Mixed and inhomogeneous expression profile of Th1/Th2 related cytokines detected by cytometric bead array in the saliva of patients with oral lichen planus. Oral. Surg. Oral. Med. Oral. Pathol. Oral. Radiol. 126, 142-151 (2018).

62. Li, Y. et al. Salivary mycobiome dysbiosis and its potential impact on bacteriome shifts and host immunity in oral lichen planus. Int J. Oral. Sci. 11, 13 (2019).

63. Wang, K. et al. Preliminary analysis of salivary microbiome and their potential roles in oral lichen planus. Sci. Rep. 6, 22943 (2016).

64. Chen, W. et al. Porphyromonas gingivalis impairs oral epithelial barrier through targeting GRHL2. J. Dent. Res. 98, 1150-1158 (2019).

65. Bombeccari, G. P., Gianni, A. B. \& Spadari, F. Oral Candida colonization and oral lichen planus. Oral. Dis. 23, 1009-1010 (2017).

66. Gladiator, A., Wangler, N., Trautwein-Weidner, K. \& LeibundGut-Landmann, S. Cutting edge: IL-17-secreting innate lymphoid cells are essential for host defense against fungal infection. J. Immunol. 190, 521-525 (2013).

67. Flores-Hidalgo, A., Murrah, V., Fedoriw, Y. \& Padilla, R. J. Relationship of infiltrating intraepithelial $\mathrm{T}$ lymphocytes in the diagnosis of oral lichen planus versus oral epithelial dysplasia: a pilot study. Oral. Surg. Oral. Med. Oral. Pathol. Oral. Radiol. 127, e123-e135 (2019).

68. Enomoto, A. et al. Intraepithelial $C D 8^{+}$lymphocytes as a predictive diagnostic biomarker for the remission of oral lichen planus. Hum. Pathol. 74, 43-53 (2018).

69. Wang, $\mathrm{H}$. et al. Oral lichen planus may enhance the expression of Th17associated cytokines in local lesions of chronic periodontitis. Clin. Oral Investig. 18, 1647-1654 (2014)

70. Xie, S. X., Ding, L., Xiong, Z. G. \& Zhu, S. G. Implications of Th1 and Th17 cells in pathogenesis of oral lichen planus. J. Huazhong Univ. Sci. Med. 32, 451-457 (2012).

71. Danielsson, K., Ebrahimi, M., Nylander, E., Wahlin, Y. B. \& Nylander, K. Alterations in factors involved in differentiation and barrier function in the epithelium in oral and genital lichen planus. Acta Derm. Venereol. 97, 214-218 (2017).

72. Danielsson, K. et al. Genes involved in epithelial differentiation and development are differentially expressed in oral and genital lichen planus epithelium compared to normal epithelium. Acta Derm.Venereol. 94, 526-530 (2014).

73. Sridevi, U., Jain, A., Nagalaxmi, V., Kumar, U. V. \& Goyal, S. Expression of Ecadherin in normal oral mucosa, in oral precancerous lesions and in oral carcinomas. Eur. J. Dent. 9, 364-372 (2015).

74. Silva, B. S. D., Yamamoto-Silva, F. P., Pontes, H. A. R. \& Pinto, D. D. E-cadherin downregulation and Twist overexpression since early stages of oral carcinogenesis. J. Oral. Pathol. Med. 43, 125-131 (2014).

75. Du, Y. \& Li, H. Expression of E-cadherin in oral lichen planus. Exp. Ther. Med. 10, 1544-1548 (2015).

76. Hamalainen, L., Soini, Y., Pasonen-Seppanen, S. \& Siponen, M. Alterations in the expression of EMT-related proteins claudin-1, claudin-4 and claudin-7, E-cadherin, TWIST1 and ZEB1 in oral lichen planus. J. Oral. Pathol. Med. 48, 735-744 (2019).

77. Amorim Dos Santos, J. et al. Laser therapy for recurrent aphthous stomatitis: an overview. Clin. Oral. Investig. 24, 37-45 (2020).

78. Cui, R. Z., Bruce, A. J. \& Rogers, R. S. 3rd Recurrent aphthous stomatitis. Clin. Dermatol. 34, 475-481 (2016).

79. Yang, Z. et al. Comparison of microbiomes in ulcerative and normal mucosa of recurrent aphthous stomatitis (RAS)-affected patients. Bmc Oral. Health 20, 128 (2020).
80. Tecco, S. et al. The association between minor recurrent aphthous stomatitis (RAS), children's poor oral condition, and underlying negative psychosocial habits and attitudes towards oral hygiene. BMC Pediatr. 18, 136 (2018).

81. Slebioda, Z., Szponar, E. \& Kowalska, A. Etiopathogenesis of recurrent aphthous stomatitis and the role of immunologic aspects: literature review. Arch. Immunol. Ther. Exp. 62, 205-215 (2014).

82. Kim, Y. J. et al. Mucosal and salivary microbiota associated with recurrent aphthous stomatitis. BMC Microbiol. 16, 57 (2016).

83. Akintoye, S.O. \& Greenberg, M.S. Recurrent aphthous stomatitis. Dent. Clin. N. Am. 58, 281-297 (2014).

84. ElAoud, S. et al. Beyond human leukocyte antigen class I antigens: hereditary hemochromatosis gene mutations in recurrent aphthous oral ulcers and behcet disease in the south of tunisia. Med. Princ. Pract. 26, 427-432 (2017).

85. Sun, A., Chia, J. S. \& Chiang, C. P. Increased proliferative response of peripheral blood mononuclear cells and T cells to Streptococcus mutans and glucosyltransferase $D$ antigens in the exacerbation stage of recurrent aphthous ulcerations. J. Formos. Med. Assoc. 101, 560-566 (2002).

86. Ruan, H. H. et al. Frequencies of abnormal humoral and cellular immune component levels in peripheral blood of patients with recurrent aphthous ulceration. J. Dent. Sci. 13, 124-130 (2018).

87. Cukrowska, B. et al. Intestinal epithelium, intraepithelial lymphocytes and the gut microbiota - key players in the pathogenesis of celiac disease. World $\mathrm{J}$. Gastroenterol. 23, 7505-7518 (2017).

88. Gunhan, O. et al. Oral epithelial barrier function and the role of nuclear factor kappa-beta pathway in the pathogenesis of aphthous ulceration. Turk. J. Gastroenterol. 24, 508-514 (2013).

89. Moutsopoulos, N. M. \& Konkel, J. E. Tissue specific immunity at the oral mucosal barrier. Trends Immunol. 39, 276-287 (2018).

90. Mason, M. R., Chambers, S., Dabdoub, S. M., Thikkurissy, S. \& Kumar, P. S. Characterizing oral microbial communities across dentition states and colonization niches. Microbiome 6, 67 (2018).

91. Tommasi, A. F. Reccurent aphthous ulceration-occurrence variation in relation to the smoking habit and its intensity. Rev. Fac. Odontol. Sao Paulo 15, 1-8 (1977).

92. Rivera-Hidalgo, F., Shulman, J. D. \& Beach, M. M. The association of tobacco and other factors with recurrent aphthous stomatitis in an US adult population. Oral. Dis. 10, 335-345 (2004).

93. Parssinen, M., Jasberg, H., Mikkonen, J. J. W. \& Kullaa, A. M. Oral mucosal pellicle as an immune protection against micro-organisms in patients with recurrent aphthous stomatitis: a hypothesis. Med. Hypotheses 146, 110449 (2021).

94. Govindarajan, B. et al. A metalloproteinase secreted by Streptococcus pneumoniae removes membrane mucin MUC16 from the epithelial glycocalyx barrier. PLOS ONE 7, e32418 (2012).

95. Lee, Y. H., Kim, Y. Y., Chang, J. Y. \& Kho, H. S. Changes in oral mucosal MUC1 expression and salivary hormones throughout the menstrual cycle. Oral. Dis. 21, 962-968 (2015).

96. Zad, M., Flowers, S. A., Bankvall, M., Jontell, M. \& Karlsson, N. G. Salivary mucin MUC7 oligosaccharides in patients with recurrent aphthous stomatitis. Clin. Oral. Investig. 19, 2147-2152 (2015).

97. Hannig, C., Hannig, M., Kensche, A. \& Carpenter, G. The mucosal pellicle - an underestimated factor in oral physiology. Arch. Oral. Biol. 80, 144-152 (2017).

98. Subramanyam, R. V. Occurrence of recurrent aphthous stomatitis only on lining mucosa and its relationship to smoking-a possible hypothesis. Med. Hypotheses 77, 185-187 (2011).

99. Johnson, N. W., Jayasekara, P. \& Amarasinghe, A. A. Squamous cell carcinoma and precursor lesions of the oral cavity: epidemiology and aetiology. Periodontology 2000. 57, 19-37 (2011).

100. Roi, A. et al. The challenges of OSCC diagnosis: salivary cytokines as potential biomarkers. J. Clin. Med. 9, E2866 (2020).

101. Liu, W. et al. Oral cancer development in patients with leukoplakiaclinicopathological factors affecting outcome. PLOS ONE 7, e34773 (2012).

102. Amer, A., Galvin, S., Healy, C. M. \& Moran, G. P. The microbiome of potentially malignant oral leukoplakia exhibits enrichment for Fusobacterium, Leptotrichia, Campylobacter, and Rothia Species. Front Microbiol. 8, 2391 (2017).

103. Hu, X., Zhang, Q., Hua, H. \& Chen, F. Changes in the salivary microbiota of oral leukoplakia and oral cancer. Oral. Oncol. 56, e6-e8 (2016).

104. Hashimoto, K. et al. Changes in oral microbial profiles associated with oral squamous cell carcinoma vs leukoplakia. J. Investig. Clin. Dent. 10, e12445 (2019).

105. Zhao, H. et al. Variations in oral microbiota associated with oral cancer. Sci. Rep. 7, 11773 (2017)

106. Lim, Y. et al. The performance of an oral microbiome biomarker panel in predicting oral cavity and oropharyngeal cancers. Front Cell Infect. Microbiol. 8, 267 (2018). 
107. Warren, R. L. et al. Co-occurrence of anaerobic bacteria in colorectal carcinomas. Microbiome 1, 16 (2013).

108. Kostic, A. D. et al. Genomic analysis identifies association of Fusobacterium with colorectal carcinoma. Genome Res. 22, 292-298 (2012).

109. Castellarin, M. et al. Fusobacterium nucleatum infection is prevalent in human colorectal carcinoma. Genome Res. 22, 299-306 (2012).

110. Amer, A., Whelan, A., Al-Hebshi, N. N., Healy, C. M. \& Moran, G. P. Acetaldehyde production by Rothia mucilaginosa isolates from patients with oral leukoplakia. J. Oral. Microbiol. 12, 1743066 (2020).

111. Ahn, J., Segers, S. \& Hayes, R. B. Periodontal disease, Porphyromonas gingivalis serum antibody levels and orodigestive cancer mortality. Carcinogenesis 33, 1055-1058 (2012).

112. Wen, L. et al. Porphyromonas gingivalis promotes oral squamous cell carcinoma progression in an immune microenvironment. J. Dent. Res. 99, 666-675 (2020).

113. Meurman, J. H. \& Uittamo, J. Oral micro-organisms in the etiology of cancer. Acta Odontol. Scand. 66, 321-326 (2008).

114. Blaser, M. J. Understanding microbe-induced cancers. Cancer Prev. Res. 1, 15-20 (2008).

115. Kakabadze, M. Z., Paresishvili, T., Karalashvili, L., Chakhunashvili, D. \& Kakabadze, Z. Oral microbiota and oral cancer: review. Oncol. Rev. 14, 476 (2020).

116. Pang, $X$. et al. Microbiota, epithelium, inflammation, and TGF-beta signaling: an intricate interaction in oncogenesis. Front. Microbiol. 9, 1353 (2018).

117. Erttmann, S. F. \& Gekara, N. O. Hydrogen peroxide release by bacteria suppresses inflammasome-dependent innate immunity. Nat. Commun. 10, 3493 (2019).

118. Wang, $H$. et al. NLRP3 promotes tumor growth and metastasis in human oral squamous cell carcinoma. BMC Cancer 18, 500 (2018).

119. Huang, M. et al. Terpenoids: natural products for cancer therapy. Expert Opin. Investig. Drugs 21, 1801-1818 (2012).

120. Gomes, E. S., Schuch, V. \& Lemos, E. G. D. Biotechnology of polyketides: New breath of life for the novel antibiotic genetic pathways discovery through metagenomics. Braz. J. Microbiol. 44, 1007-1034 (2013).

121. Johnson, S. D., Levingston, C. \& Young, M. R. Premalignant oral lesion cells elicit increased cytokine production and activation of T-cells. Anticancer Res. 36, 3261-3270 (2016).

122. Omenetti, S. \& Pizarro, T. T. The Treg/Th17 axis: a dynamic balance regulated by the gut microbiome. Front. Immunol. 6, 639 (2015).

123. Ma, Y. et al. IL-6, IL-8 and TNF-alpha levels correlate with disease stage in breast cancer patients. Adv. Clin. Exp. Med. 26, 421-426 (2017).

124. Robayo, D. A. G. et al. Oral microbiota associated with oral and gastroenteric cancer. Open Microbiol. J. 14, 1-17 (2020).
125. Groeger, S., Domann, E., Gonzales, J. R., Chakraborty, T. \& Meyle, J. B7-H1 and B7DC receptors of oral squamous carcinoma cells are upregulated by Porphyromonas gingivalis. Immunobiology 216, 1302-1310 (2011).

126. Raskov, H., Burcharth, J. \& Pommergaard, H. C. Linking gut microbiota to colorectal cancer. J. Cancer 8, 3378-3395 (2017).

127. Gur, C. et al. Binding of the Fap2 protein of Fusobacterium nucleatum to human inhibitory receptor TIGIT protects tumors from immune cell attack. Immunity $\mathbf{4 2 ,}$ 344-355 (2015).

128. Santosh, A. B. \& Jones, T. J. The epithelial-mesenchymal interactions: insights into physiological and pathological aspects of oral tissues. Oncol. Rev. 8, 239 (2014).

129. Wang, H. \& Unternaehrer, J. J. Epithelial-mesenchymal transition and cancer stem cells: at the crossroads of differentiation and dedifferentiation. Dev. Dyn. 248, 10-20 (2019).

130. Kuboniwa, M. et al. $P$. gingivalis accelerates gingival epithelial cell progression through the cell cycle. Microbes Infect. 10, 122-128 (2008).

131. Whitmore, S. E. \& Lamont, R. J. Oral bacteria and cancer. PLoS Pathog. 10, e1003933 (2014).

132. Geng, F. X. et al. Persistent exposure to Porphyromonas gingivalis promotes proliferative and invasion capabilities, and tumorigenic properties of human immortalized oral epithelial cells. Front Cell Infect. Microbiol. 7, 57 (2017).

133. Chattopadhyay, I., Verma, M. \& Panda, M. Role of oral microbiome signatures in diagnosis and prognosis of oral cancer. Technol. Cancer Res. Treat. 18, 1533033819867354 (2019).

134. Al-hebshi, N. N. et al. Inflammatory bacteriome featuring Fusobacterium nucleatum and Pseudomonas aeruginosa identified in association with oral squamous cell carcinoma. Sci. Rep. 7, 1834 (2017).

135. Yaegaki, K. Oral malodorous compounds are periodontally pathogenic and carcinogenic. Jpn. Dent. Sci. Rev. 44, 100-108 (2008).

136. Cheng, R. et al. Reduced CX3CL1 secretion contributes to the susceptibility of oral leukoplakia-associated fibroblasts to Candida albicans. Front. Cell Infect. Microbiol. 6, 150 (2016).

137. Blaskewicz, C. D., Pudney, J. \& Anderson, D. J. Structure and function of intercellular junctions in human cervical and vaginal mucosal epithelia. Biol. Reprod. 85, 97-104 (2011).

138. Tsukita, S. \& Furuse, M. Overcoming barriers in the study of tight junction functions: from occludin to claudin. Genes Cells 3, 569-573 (1998).

139. Dhawan, P. et al. Claudin-1 regulates cellular transformation and metastatic behavior in colon cancer. J. Clin. Investig. 115, 1765-1776 (2005).

140. Phattarataratip, E. \& Sappayatosok, K. Expression of claudin-5, claudin-7 and occludin in oral squamous cell carcinoma and their clinico-pathological significance. J. Clin. Exp. Dent. 8, e299-e306 (2016). 